Research Paper

\title{
Plasma Brain-derived Neurotrophic Factor in Hemodialysis Patients
}

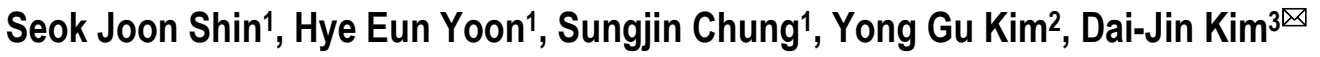

1. Division of Nephrology, Department of Internal Medicine, College of Medicine, The Catholic University of Korea, Seoul, Korea

2. Department of Psychiatry, College of Medicine, Korea University, Seoul, Korea

3. Department of Psychiatry, College of Medicine, The Catholic University of Korea, Seoul, Korea

$\triangle$ Corresponding author: Dai-Jin Kim. Department of Psychiatry, Seoul St. Mary's Hospital, College of Medicine, The Catholic University of Korea, 222 Banpo-daero, Seocho-gu, Seoul 137-701, Korea. Email: kdj922@chol.com

(C) Ivyspring International Publisher. This is an open-access article distributed under the terms of the Creative Commons License (http://creativecommons.org/ licenses/by-nc-nd/3.0/). Reproduction is permitted for personal, noncommercial use, provided that the article is in whole, unmodified, and properly cited.

Received: 2012.08.16; Accepted: 2012.10.15; Published: 2012.10.24

\begin{abstract}
Objective: Brain-derived neurotrophic factor (BDNF) is a neurotrophin which modulates neuronal survival and proliferation. Recently, plasma BDNF is associated with inflammatory conditions. The present study was to investigate the changes of the plasma BDNF level in hemodialysis patients and to evaluate the relationship between the plasma BDNF and uremic inflammation.

Method: We measured the plasma BDNF, serum IL-2, IL-4, IFN- $\gamma$ and TNF- $\alpha$ in hemodialysis patients and control subjects. In addition, we investigated the associations of the plasma BDNF with clinical or biochemical parameters.

Results: The plasma BDNF level was significantly higher in hemodialysis patients than in the control subjects (median, 312.3 vs. $630.3 \mathrm{pg} / \mathrm{ml}, p<0.05$ ). In subgroup analysis, hemodialysis patients with diabetes showed higher values of the plasma BDNF than the patients without diabetes. However, there were no significant differences in age and gender subgroups of the hemodialysis patients. The logBDNF was positively correlated with hs-CRP and IFN-y, and negatively correlated with serum albumin. In multivariate regression analysis, the logBDNF was independently associated with the presence of diabetes $(\beta$-coefficient $=0.399, p=0.04 \mathrm{I})$ and IFN-Y $(\beta$-coefficient $=0.538, p=0.003)$.

Conclusion: The plasma BDNF may increase in hemodialysis patients, and more prominently in the patients with diabetes. Furthermore, the plasma BDNF might reflect inflammatory condition in hemodialysis patients.
\end{abstract}

Key words: Brain-derived neurotrophic factor, Cytokine, Hemodialysis, Uremia, Inflammation

\section{Introduction}

Brain-derived neurotrophic factor (BDNF) is a member of the nerve growth factor family and it exerts its effects by binding to the tropomyosin kinase B receptor. Among the neurotrophins, BDNF is the most abundant and widely distributed in the central nervous system. BDNF is a basic dimeric protein, and its subunit is a $13.5 \mathrm{kDa}$ (119 amino acids) molecule. It is an essential mediator of neuronal activity and synaptic plasticity in both the central and peripheral nerv- ous systems, and it enhances the growth and maintenance of several neuronal systems by serving as a neurotransmitter modulator (1). It is well known that the BDNF expression increases following an insult to the central nervous system (CNS). Although many studies have demonstrated a relevant role of BDNF for regulating neuroprotection, recent findings have suggested that changes of plasma BDNF level, regardless of nervous system, are also associated with 
several inflammatory or metabolic derangement conditions (2-4).

Decrease of renal function induces accumulations of circulating cytokines, advanced glycation end product and pro-oxidants, which contributes to the pro-inflammatory milieu in patients with chronic kidney disease. Uremic inflammation is associated with various adverse outcomes in hemodialysis (HD) patients, such as cardiovascular disease, malnutrition and neurological disorders. While many studies have investigated inflammatory cytokines as a reflection of uremic condition in HD patients, there is no information about the peripheral changes of BDNF in patients undergoing HD as far as we are aware.

In this cross-sectional study, we investigated the changes of the plasma BDNF levels in HD patients and evaluated the potential correlations between this peptide and selected parameters of uremic inflammation.

\section{Materials and Methods}

\section{Study populations}

Using a cross-sectional design, we studied 60 randomly selected, stable uremic patients who were undergoing $\mathrm{HD}$ for more than 6 months and 30 healthy control subjects who were matched for age and sex. All those joined in this study provided us a written informed consent. This study was approved by the hospital ethics committee (Incheon St. Mary's Hospital, The Catholic University of Korea). Patients with known hypersensitivity to the dialysis membrane, signs of infection, malignancy, severe liver disease, autoimmune disease, previous history of renal transplantation, treatment with drugs known to interfere with the immune system were excluded.

\section{Plasma Brain-derived Neurotrophic Factor and Inflammatory cytokines}

Following an overnight fast, blood samples were collected from both HD patients and control subjects. For the HD patients, the samples were obtained from the arterial side at the time of needling before the dialysis session. Fasting blood was withdrawn into a lithium heparin vacuum tube and immediately centrifuged at 3,800 rpm for 10 minutes. The plasma was stored at $-70^{\circ} \mathrm{C}$ until it was thawed for assay. Human plasma BDNF was assayed using the DuoSet ELISA Development System (Catalog number: DY248, R\&D Systems, UK). All the assays were performed in duplicate using the manufacturer's recommended buffers, diluents and substrates. All assays had a detection range from 20 to $4000 \mathrm{pg} / \mathrm{ml}$. The intra-assay and inter-assay coefficients of variation were $5.4 \%$ and
$9.8 \%$, respectively. The concentrations of the samples in each plate were calculated according to each standard curve and the dilution factor. The cytokine assays for human serum IL-2, IL-4, tumor necrosis factor- $\alpha$ (TNF- $\alpha)$ and interferon- $\gamma($ IFN- $\gamma)$ were performed using the highly sensitive HS-ELISA Development System from R\&D Systems, Abingdon, UK. All the assays were performed according to the manufacturer's instruction. The intra- and inter-assay coefficients of variation were 5.6 and $7.08 \%$, respectively, for IL-2, IL-4, and TNF-a. The intra- and inter-assay coefficients of variation were 6.7 and $9.2 \%$ for IFN- $\gamma(5)$.

\section{Clinical and Biochemical Parameters}

The presence of diabetes, body mass index (BMI), dialysis duration and dialysis adequacy (Kt/Vurea) were assessed in the HD patients. We also measured hemoglobin, hematocrit, platelet count, blood urea nitrogen, creatinine, serum albumin, lipid profiles, calcium, phosphorus, intact parathyroid hormone (iPTH), high sensitivity C-reactive protein (hs-CRP, normal value $<3.0 \mathrm{mg} / \mathrm{L}$ ), ferritin and fibrinogen in these patients.

\section{Statistical Analyses}

The data with continuous variables and a normal distribution were presented as means \pm standard error, and those without a normal distribution were presented as the median with ranges. The Kolmogorov-Smirnov test showed that the BDNF values did not fit a standard distribution curve. Therefore, statistical analyses were performed using an appropriate non-parametric test, the Mann-Whitney $U$-test. The chi-square test was used to determine the differences in categorical variables. The correlation between selected variables was determined by Spearman's correlation analysis. The relationship between BDNF levels and other clinical or biochemical parameters was also analyzed by multiple regression analysis. The statistical significance level was set at $p<0.05$. SPSS 15.0 software was used for these analyses.

\section{Results}

The plasma BDNF level was significantly higher in HD patients than in control subjects (median 630.3, range 76.4-1435.0 vs. 312.4, 78.7-969.9 pg/ml, respectively, $p<0.05$ ) (Figure 1). Serum IL-2, IL-4, TNF-a and IFN- $\gamma$ also showed higher values in the HD group than in the control group $(p<0.05)$. There were no significant differences in age, gender and BMI between the HD group and the control group (Table 1). The baseline characteristics of the HD patients are summarized in Table 2. Among the 60 HD patients, 26 
$(43.3 \%)$ had diabetes. The median duration of dialysis was 43.5 months (range, 7-240 months). We classified HD patients into subgroups according to age, gender and the presence of diabetes, and compared the plasma BDNF levels between subgroups. There were no significant differences in the plasma BDNF level in subgroup analysis according to age ( $\geq 60$ years, median 592.7, range 90.5-1380.0 pg/ml; $\leq 60$ years, median 635.7, range 76.4-1435.0 pg/ml; $p=0.83$ ) and gender (male, median 512.0, range 76.4-1435.0 pg/ml; female, median 625.3, range $90.5-1412.6 \mathrm{pg} / \mathrm{ml} ; p=$ 0.40). However, the plasma BDNF level of diabetic HD patients showed higher value compared to those of non-diabetic HD patients and control subjects (diabetic HD patients, median 822.4, range 90.5-1435.0 $\mathrm{pg} / \mathrm{ml}$; non-diabetic patients, median 418.7 , range 102.4-835.0 pg/ml; control subjects, median, 312.4,

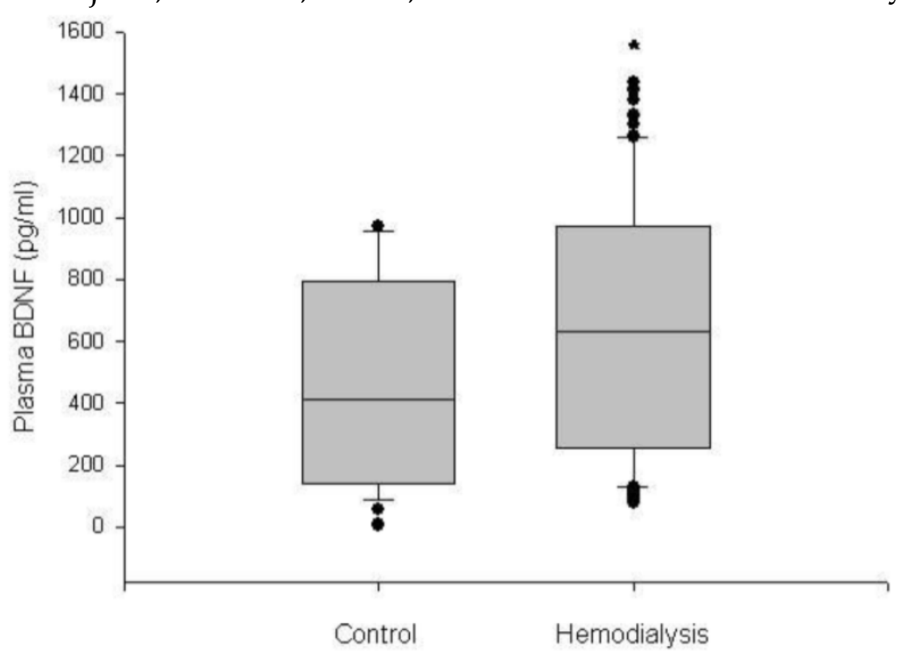

Figure I. Box plot diagrams of plasma BDNF levels in the control subjects and hemodialysis patients. Boxplots display the median, quartiles and extremes. ${ }^{*} p<0.05$ vs. control group.

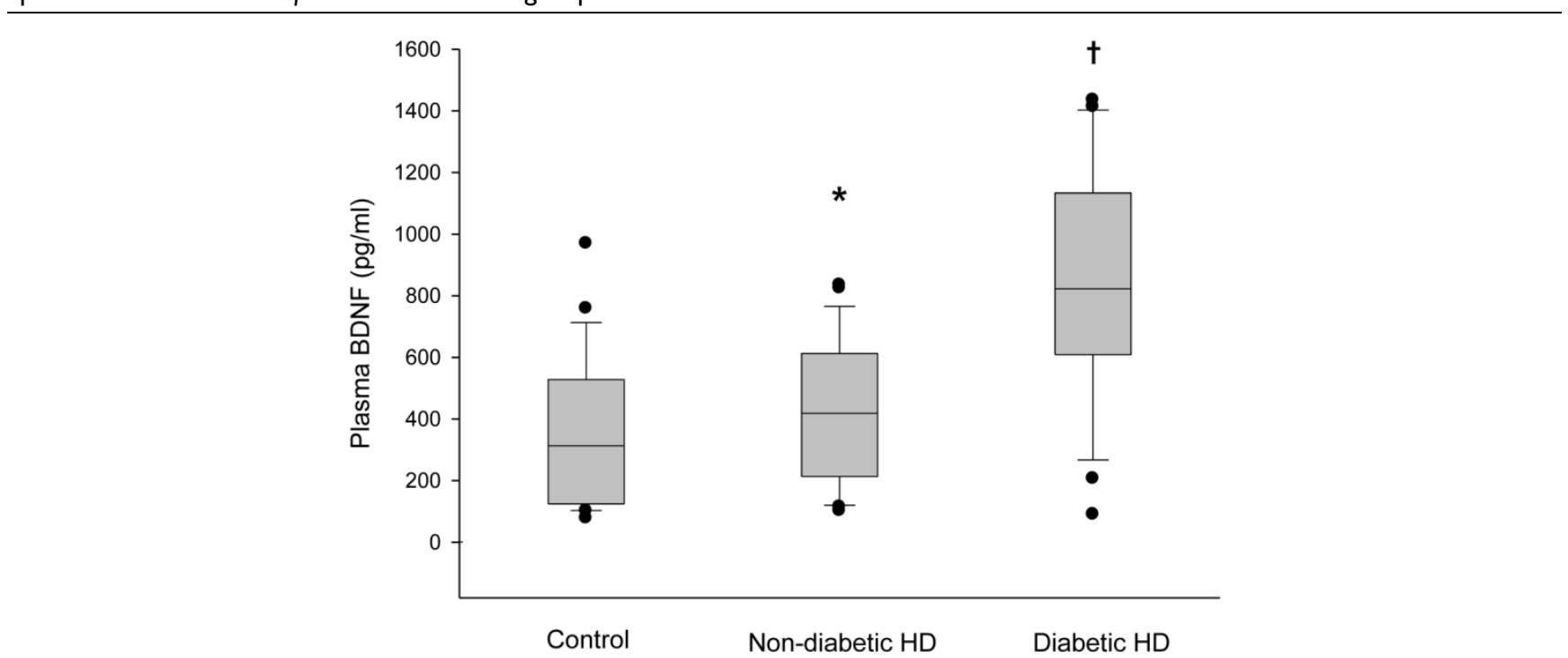

Figure 2. Box plot diagrams of plasma BDNF levels in the control subjects, non-diabetic hemodialysis and diabetic hemodialysis patients. Box plots display the median, quartiles and extremes. ${ }^{*} p<0.05$ vs. control group, ${ }^{\dagger} p<0.05$ vs. other groups. range 78.7-969.9 pg/ml; $p<0.01$ ). Furthermore, non-diabetic HD patients showed slightly, but significantly higher plasma BDNF value than control subjects $(p=0.022$; Figure 2). Correlation analysis was performed to examine the relationships between $\log B D N F$ and the clinical or biochemical parameters in the HD group. The logBDNF was positively correlated with hs-CRP $(\mathrm{r}=0.409, p<0.01)$ and IFN-Y $(\mathrm{r}=$ $0.551, p<0.01$ ), and it was negatively correlated with serum albumin $(\mathrm{r}=-0.408, p<0.01)$. There was no significant association between $\log B D N F$ and other clinical or biochemical parameters. In multivariate regression analysis, $\log B D N F$ was independently related to the presence of diabetes $(\beta$-coefficient $=0.399$, $p=0.041)$ and IFN- $\gamma(\beta$-coefficient $=0.538, p=0.003)$ after adjustment for age, sex, BMI, dialysis duration, serum albumin, CRP and cytokines (Table 3). 
Table I. Plasma BDNF and serum cytokines of study subjects.

\begin{tabular}{lll}
\hline Blood level & Control $(\mathrm{n}=30)$ & HD $(\mathrm{n}=60)$ \\
\hline Age $($ years $)$ & $43.2 \pm 12.1$ & $43.8 \pm 7.7$ \\
Male, $n(\%)$ & $13(44.5)$ & $27(45.5)$ \\
BMI $\left(\mathrm{kg} / \mathrm{m}^{2}\right)$ & $22.8 \pm 0.9$ & $22.1 \pm 0.4$ \\
BDNF $(\mathrm{pg} / \mathrm{ml})$ & $312.4(78.7-969.9)$ & $630.3(76.4-1,435.0)^{*}$ \\
IL-2 $(\mathrm{pg} / \mathrm{ml})$ & $278.9 \pm 11.0$ & $761.8 \pm 18.4^{*}$ \\
IL-4 $(\mathrm{pg} / \mathrm{ml})$ & $367.9 \pm 44.3$ & $789.6 \pm 44.8^{*}$ \\
IFN-ץ $(\mathrm{pg} / \mathrm{ml})$ & $491.9 \pm 27.5$ & $748.6 \pm 28.5^{*}$ \\
TNF- $\mathrm{\alpha}(\mathrm{pg} / \mathrm{ml})$ & $322.1 \pm 38.3$ & $457.0 \pm 20.2^{*}$ \\
\hline
\end{tabular}

*Significantly $(\mathrm{P}<0.05)$ different from the controls. Values are expressed as mean \pm standard error, median (range). HD: hemodialysis group; BMI: body mass index.

Table 2. Clinical characteristics of hemodialysis patients.

\begin{tabular}{llll}
\hline Baseline characteristics & HD $(\mathrm{n}=60)$ & Baseline characteristics & HD $(\mathrm{n}=60)$ \\
\hline Diabetes (yes, \%) & $26(43.3)$ & Serum albumin $(\mathrm{g} / \mathrm{dl})$ & $4.2 \pm 0.4$ \\
Duration of dialysis $(\mathrm{mo})$ & $43.5(7-240)$ & Total cholesterol $(\mathrm{mg} / \mathrm{dl})$ & $169.8 \pm 34.6$ \\
Kt/Vurea & $1.21 \pm 0.21$ & Triglyceride $(\mathrm{mg} / \mathrm{dl})$ & $132.0 \pm 67.5$ \\
Hemoglobin $(\mathrm{g} / \mathrm{dl})$ & $10.1 \pm 2.3$ & Calcium $\times$ Phosphorus & $44.4 \pm 2.3$ \\
Hematocrit $(\%)$ & $30.4 \pm 5.9$ & iPTH $(\mathrm{pg} / \mathrm{ml})$ & $43.6(2.6-1,869.0)$ \\
Platelet $\left(\times 10^{3} / \mathrm{mm}^{3}\right)$ & $197 \pm 8.7$ & hs-CRP $(\mathrm{mg} / \mathrm{L})$ & $4.0 \pm 7.1$ \\
Blood urea nitrogen $(\mathrm{mg} / \mathrm{dl})$ & $60.3 \pm 13.8$ & Ferritin $(\mathrm{mg} / \mathrm{dl})$ & $175(25-1,156)$ \\
Creatinine $(\mathrm{mg} / \mathrm{dl})$ & $10.0 \pm 2.9$ & Fibrinogen $(\mathrm{mg} / \mathrm{L})$ & $333.1 \pm 117.8$
\end{tabular}

Values are expressed as mean \pm standard error, median (range). HD: hemodialysis group; iPTH: intact parathyroid hormone; hs-CRP: high sensitivity C-reactive protein.

Table 3. Association between logBDNF and the presence of diabetes or IFN-y in hemodialysis patients; Multivariate regression analysis.

\begin{tabular}{lllll}
\hline & Diabetes & & IFN- $\gamma$ & \\
\hline & $\beta$-coefficient & $P$ & $\beta$-coefficient & $P$ \\
$\operatorname{logBDNF}$ & 0.399 & 0.041 & 0.538 & 0.003
\end{tabular}

Data are expressed as standardized regression coefficient $(\beta)$ and $p$ value. Diabetes was set as a dichotomous variable. Diabetes mellitus and non-diabetes mellitus were assigned values of 1 and 0 , respectively. Also included in the model were age, sex, body mass index, dialysis duration, serum albumin, hs-CRP and cytokines.

\section{Discussion}

According to many other studies, BDNF has been shown to protect the CNS under a variety of conditions. Previous work demonstrated that patients with fibromyalgia and depression have increased serum BDNF concentrations (6). It has also been shown that plasma BDNF concentrations were elevated in drug users or in patients with chronic stress $(7,8)$. Recently, apart from nervous systems, there are several reports showing the associations of plasma BDNF with systemic or peripheral inflammatory conditions, such as diabetes, acute coronary syndrome, atherosclerosis or rheumatoid arthritis (2-4).

In this study, we investigated for the first time the changes of the plasma BDNF level in uremic condition and the correlations between BDNF and clinical or biochemical parameters in HD patients. We found that the plasma BDNF level was significantly elevated in patients who were undergoing HD compared to the control subjects. The plasma BDNF was removed during dialysis session. In our study, the average plasma BDNF clearance of a single HD session, which is defined as the pre-HD blood levels minus the post-HD blood levels divided by the pre-HD blood levels, was $\sim 19.4 \%$, and the median post-HD plasma BDNF level was $508.1 \mathrm{pg} / \mathrm{ml}$ (range, 25.1-1327.0 $\mathrm{pg} / \mathrm{ml}$ ). The post-HD plasma BDNF level was significantly higher than that of the controls $(p<0.05)$. However, there was no correlation between the post-HD plasma BDNF and the clinical or biochemical parameters including cytokines (data not shown).

In the present study, the increase of the BDNF to be more marked in the HD patients with diabetes. 
Previous animal research demonstrated that subcutaneous administration of BDNF reduced food intake and ameliorated impaired glucose tolerance in diet-induced obesity mice (9). Furthermore, systemic BDNF treatment normalized fasting blood glucose, hemoglobin $\mathrm{A}_{1 \mathrm{c}}$ and lipid profiles $(10,11)$. BDNF heterozygous knockout (BDNF+/-) mice showed obesity and insulin resistance (12). Therefore, in the present study, it is possible that plasma BDNF may increase in HD patients with diabetes to compensate hyperinsulinemia and insulin resistance. However, there is a controversy as to whether the serum BDNF level is higher in diabetics than in non-diabetics who are not undergoing dialysis treatment. A previous study showed that serum BDNF level was higher in newly diagnosed type 2 diabetic patients than in normal controls (2). On the other hand, Fujinami et al. reported that serum BDNF levels were significantly lower in patients with advanced type 2 diabetes compared to control subjects (13). In our results, most of diabetes in dialysis group were not newly diagnosed, and might be more complicated diabetes. Therefore, the presence of diabetes alone could not explain the elevated level of the plasma BDNF in the present study. Our results also showed that the plasma BDNF level in non-diabetic HD patients was higher than that of control subjects. There may be many other factors including uremic inflammation to increase the plasma BDNF level in diabetes of the HD patients. Further investigations are needed to clarify these points.

There were no differences of plasma BDNF level according to age or gender subgroups in our HD patients. Conflicting results exist as regards the changes of BDNF associated with age or gender differences. A recent report showed that a significant age-related increase of the plasma BDNF level in Down's syndrome patients (14). However, there are also reports showing age-related decrease of the serum BDNF level or no significant difference of the serum BDNF in age $(6,13)$. In the gender aspect, a previous report showed that BDNF levels were significantly higher in females than in males with type 2 diabetes (2). In contrast, Laske et al. revealed no significant gender difference of the serum BDNF in fibromyalgia patients (6). It seems that gender or age-related serum BDNF results could differ according to underlying disease and study population.

It is well known that the uremic status is a constant inflammatory condition. Recent studies demonstrated that pro-inflammatory cytokines, such as IL-1, IL-6, TNF- $\alpha$ and IFN- $\gamma$ showed higher value. Anti-inflammatory cytokine levels including IL-2, IL-4, IL-5 and IL-12 were decreased in dialysis patients (15).
In the present study, the serum cytokine levels were increased in the HD patients but not in controls. In addition, the HD patients showed a significantly positive relationship between the plasma BDNF and the serum IFN- $\gamma$ in multivariate regression analysis. The association of the plasma BDNF with inflammatory conditions has been reported. Increases of BDNF expression in inflamed bladder tissues and in airway epithelium during allergic airway inflammation have been demonstrated $(16,17)$. Furthermore, Grimsholm et al reported that plasma BDNF level in rheumatoid arthritis patients showed higher values than in healthy controls, and anti-TNF treatment lead to a decrease in the plasma BDNF level (4). It is unclear that higher plasma BDNF in the HD patients was associated with uremic inflammation. It has been shown that activated antigen-specific $\mathrm{T}$ cells, $\mathrm{B}$ cells, and monocytes produced BDNF (18). Uremic inflammatory state is characterized by enhanced immune system which involves both $\mathrm{T}$ lymphocytes and monocytes (19). Therefore, it is postulated that chronic inflammatory condition and enhanced immune system is associated with the production of BDNF, which may explain the reason that the plasma BDNF level in non-diabetic HD patients was higher compared to that of control subjects in this study.

BDNF is found in both human serum and plasma. Serum contains platelets, and it has been well established that large amount of BDNF is stored in platelets (20). It was previously reported that serum BDNF level was found to be 200-fold higher than plasma level (21). Thrombin, collagen, the $\mathrm{Ca}^{++}$ionophore A23187 and shear stress stimulated a rapid release of BDNF from platelets (20). Thus, the difference between serum and plasma levels of BDNF could explain the amount of BDNF stored in platelets, and plasma BDNF may reflect circulating levels rather than the levels stored in platelets (22). Only a few data are available on plasma BDNF levels and it is difficult to determine the significance of BDNF of plasma according to disease state. Nofuji et al. recently reported that the serum BDNF concentration was found to significantly change according to exercise, however, the plasma BDNF level was not (23). On the other hand, Piccinni et al. showed in their study with depressed patients that while the plasma BDNF levels increased in parallel with the clinical improvement after one month antidepressant treatment, they could not detect any significant change in serum BDNF levels in spite of the treatment (24). In the future, studies will be required to determine the action and regulation of plasma BDNF in peripheral blood according to disease state.

There are some limitations in this study. First, 
this is a cross sectional study, therefore, longitudinal observation is needed to assess the relationship between this peptide with uremic inflammatory conditions and clinical outcomes. Second, this study is limited by its small sample size. Further study with a large dialysis population would add further information about the role of BDNF as markers of inflammation and malnutrition including hs-CRP and serum albumin. Third, in this study, we measured only the plasma levels of BDNF, and this was the major limitation of the study. The source of plasma BDNF and its biological activity were not investigated in the present study. Future studies are needed to determine which source of BDNF - platelets, plasma, serum, or whole blood - provides the most reliable biological marker of patients with HD. Finally, we did not provide data on the assessment of nervous system functions in the patients and the other neurotrophins such as nerve growth factor or neurotrophin-3. The evaluation of these parameters in the HD patients may help to explain the change of the plasma BDNF level in uremic condition.

In conclusion, the plasma BDNF level showed higher values in the HD patients, and the presence of diabetes seemed to be associated with the higher value of the plasma BDNF in the patients. In addition, the plasma BDNF level was found to be positively correlated with inflammatory cytokine in these patients, which suggests that plasma BDNF might reflect the uremic inflammatory condition in the patients undergoing HD.

\section{Competing Interests}

The authors have declared that no competing interest exists.

\section{References}

1. Barbacid M. Neurotrophic factors and their receptors. Curr Opin Cell Biol. 1995; 7: 148-55.

2. Suwa M, Kishimoto H, Nofuji Y, et al. Serum brain-derived neurotrophic factor level is increased and associated with obesity in newly diagnosed female patients with type 2 diabetes mellitus. Metabolism. 2006; 55: 852-7.

3. Manni L, Nikolova V, Vyagova D, et al. Reduced plasma levels of NGF and BDNF in patients with acute coronary syndromes. Int J Cardiol. 2005; 102: 169-71.

4. Grimsholm O, Rantapää-Dahlqvist $S$, Dalén $T$, et al. BDNF in RA: Downregulated in plasma following anti-TNF treatment but no correlation with inflammatory parameters. Clin Rheumatol. 2008; 27: 1289-97.

5. Kim YK, Myint AM, Verkerk R, et al. Cytokine changes and tryptophan metabolites in medication-naïve and medication-free schizophrenic patients. Neuropsychobiology. 2009; 59: 123-9.

6. Laske C, Stransky E, Eschweiler GW, et al. Increased BDNF serum concentration in fibromyalgia with or without depression or antidepressants. J Psychiatr Res. 2007; 41: 600-5.

7. Dagnino-Subiabre A, Zepeda-Carreno R, Diaz-Veliz G, et al. Chronic stress induces upregulation of brain-derived neurotrophic factor (BDNF) mRNA and integrin alpha5 expression in the rat pineal gland. Brain Res. 2006; 1086: 27-34.
8. Kim DJ, Roh S, Kim Y, et al. High concentrations of plasma brain-derived neurotrophic factor in methamphetamine users. Neurosci Lett. 2005; 388: 112-5.

9. Nakagawa T, Ogawa Y, Ebihara K, et al. Anti-obesity and anti-diabetic effects of brain-derived neurotrophic factor in rodent models of leptin resistance. Int J Obes Relat Metab Disord. 2003; 27: 557-65.

10. Tonra JR, Ono M, Liu X, et al. Brain-derived neurotrophic factor improves blood glucose control and alleviates fasting hyperglycemia in C57BLKS-Lepr(db)/lepr(db) mice. Diabetes. 1999; 48: 588-94.

11. Tsuchida A, Nonomura $T$, Nakagawa $T$, et al. Brain-derived neurotrophic factor ameliorates lipid metabolism in diabetic mice. Diabetes Obes Metab. 2002; 4: 262-9.

12. Duan W, Guo Z, Jiang H, et al. Reversal of behavioral and metabolic abnormalities, and insulin resistance syndrome, by dietary restriction in mice deficient in brain-derived neurotrophic factor. Endocrinology. 2003; 144: 2446-53.

13. Fujinami A, Ohta $K$, Obayashi $H$, et al. Serum brain-derived neurotrophic factor in patients with type 2 diabetes mellitus: Relationship to glucose metabolism and biomarkers of insulin resistance. Clin Biochem. 2008; 41: 812-7.

14. Dogliotti G, Galliera E, Licastro F, et al. Age-related changes in plasma levels of BDNF in Down syndrome patients. Immun Ageing. 2010; 7: 2.

15. Cohen SD, Phillips TM, Khetpal P, et al. Cytokine patterns and survival in haemodialysis patients. Nephrol Dial Transplant. 2010; 25: 1239-43.

16. Oddiah D, Anand P, McMahon SB, et al. Rapid increase of NGF, BDNF and NT-3 mRNAs in inflamed bladder. Neuroreport. 1998; 9: 1455-8.

17. Hahn C, Islamian AP, Renz H, et al. Airway epithelial cells produce neurotrophins and promote the survival of eosinophils during allergic airway inflammation. J Allergy Clin Immunol. 2006; 117: 787-94.

18. Kerschensteiner M, Gallmeier E, Behrens L, et al. Activated human $\mathrm{T}$ cells, B cells, and monocytes produce brain-derived neurotrophic factor in vitro and in inflammatory brain lesions: a neuroprotective role of inflammation? J Exp Med. 1999; 189: 865-70.

19. Pereira R, Costa E, Gonçalves M, et al. Neutrophil and monocyte activation in chronic kidney disease patients under hemodialysis and its relationship with resistance to recombinant human erythropoietin and to the hemodialysis procedure. Hemodial Int. 2010; 14: 295-301.

20. Fujimura $\mathrm{H}$, Altar CA, Chen R, et al. Brain-derived neurotrophic factor is stored in human platelets and released by agonist stimulation. Thromb Haemost. 2002; 87: 728-34.

21. Rosenfeld RD, Zeni L, Haniu M, et al. Purification and identification of brain-derived neurotrophic factor from human serum. Protein Expr Purif. 1995; 6: 465-71.

22. Chula BL, Choia I, Kim YK, et al. Relation between plasma brain-derived neurotrophic factor and nerve growth factor in the male patients with alcohol dependence. Alcohol. 2009; 43: 265-9.

23. Nofuji Y, Suwa M, Sasaki H, et al. Different circulating brain-derived neurotrophic factor responses to acute exercise between physically active and sedentary subjects. J Sports Sci Med. 2012; 11: 83-8.

24. Piccinni A, Marazziti D, Catena $M$, et al. Plasma and serum brain-derived neurotrophic factor (BDNF) in depressed patients during 1 year of antidepressant treatments. J Affect Disord. 2008; 105: 279-83. 